\title{
Pontos de Referência para Navegação por Motoristas com Deficiência na Visão de Cores
}

\author{
Landmarks for Navigation by Drives' with Color Vision Deficiency
}

\author{
Edmur Azevedo Pugliesi ${ }^{1}$ \\ Aline Moretti ${ }^{2}$ \\ Ana Paula Marques Ramos ${ }^{3}$ \\ Vilma Mayumi Tachibana ${ }^{4}$
}

\begin{abstract}
RESUMO
O objetivo deste estudo é identificar pontos de referência que sejam potenciais para navegação em automóvel, considerando motoristas que possuem Deficiência na Visão de Cores - DVC. Os Sistemas de Navegação e Guia de Rota em Automóvel (SINGRA) exibem semáforos e viadutos, os quais estão entre os elementos de contexto ambiental mais utilizados pelos motoristas com Visão Normal de Cores - VNC. Um estudo empírico investigou a demanda por informação de guia de rota junto a um grupo de motoristas que possuem DVC por meio de duas técnicas de externalização de informações, esboço cartográfico e anotações verbais. Os dados coletados junto aos motoristas com DVC foram comparados entre as diferentes técnicas, e os dados coletados por meio dos esboços cartográficos originados deste estudo foram comparados com os dados coletados em um estudo anterior, o qual utilizou motoristas com VNC. Dos pontos de referência que apresentaram maior frequência de uso entre os participantes, alguns são mais importantes para guia de rota do que outros. A técnica de anotação verbal complementou a do esboço cartográfico, sugerindo que a aplicação de somente uma não permitiria encontrar resultados mais conclusivos. Viaduto e hospital foram os mais utilizados, seguido de parque e semáforo. Os atributos que definiram a qualidade dos pontos de referência são apresentados do mais valorado ao menos valorado. Os semáforos, viadutos e parques deveriam ser incluídos nos SINGRA para apoiar a tarefa de manutenção em rota de motoristas com DVC. Novos pontos de referência podem emergir de acordo com as características de cada ambiente urbano.
\end{abstract}

Recebido em agosto de 2018 Aprovado em fevereiro de 2019.

\footnotetext{
1Universidade Estadual Paulista "Júlio de Mesquita Filho". Programa de Pós-Graduação em Ciências Cartográficas. E-mail: edmur.pugliesi@unesp.br

2Universidade Estadual Paulista "Júlio de Mesquita Filho". Departamento de Planejamento Urbanismo e Ambiente. E-mail: alinemoretti@hotmail.com

3Universidade do Oeste Paulista. Departamento de Geografia. E-mail: anaramos@unoeste.br ${ }^{4}$ Universidade Estadual Paulista "Júlio de Mesquita Filho". Programa de Pós-Graduação em Ciências Cartográficas. E-mail: vilma.tachibana@unesp.br
} 
PALAVRAS-CHAVE: Sistema de Navegação e Guia de Rota em Automóvel. Pontos de Referência. Deficiência na Visão de Cores. Mapas Cognitivos.

\begin{abstract}
The objective of this study is to identify potential landmarks for in-car navigation, considering drivers who present Color Vision Deficiency - CVD. In-Car Navigation Systems (RGNS) display traffic light and bridge over road, which are among the environment context elements more used by the drivers having Normal Color Vision - NCV. An empirical study investigated the demand for route guide information with a group of drivers who have CVD by using two techniques of information externalization, map sketch and verbal note. Data collected with drivers having CVD were compared between both techniques, and data collected from map sketch, originated from this study, were compared with data collected in a previous study, which used drivers having NCV. From the landmarks that presented the highest frequency of using among the participants, some are more important for route guidance than others. Verbal notes technique complemented the map sketch, suggesting that applicating just one would not be enough to find more conclusive results. Bridge over road and hospital were the most used, followed by park and traffic light. The attributes that defined the quality of landmarks are presented from the most valued. Traffic light, bridge over road and park should be included in maps of Car Navigation Systems to aid the task of route following for drivers having CVD. New landmarks may emerge according to the characteristics of each environment.
\end{abstract}

KEYWORDS: In-Car Route Guidance and Navigation System. Landmarks. Color Vision Deficiency. Cognitive map.

$$
* * *
$$

\title{
Introdução
}

Dirigir um automóvel em uma área não familiar é uma das atividades que mais exige demanda cognitiva do motorista (PETCHENIK, 1989; De WAARD, 1996). Para apoiar as tarefas de planejamento de rota e manutenção em rota, os Sistemas de Navegação e Guia de Rota em Automóvel (SINGRA) fornecem instruções para navegação. Essas instruções estão relacionadas às demandas dos motoristas e compreendem informações de direção (local, egocêntrica e global), distância e contexto do ambiente (ALM, 1990, OBATA, DAIMON; KAWASHIMA, 1993; BURNETT, 1998; DAIMON et al., 2000; LEE; FORLIZZI; HUDSON, 2008; PAPINSKI; SCOTT; DOHERTY, 2009; PUGLIESI et al., 2014). 
As informações do contexto ambiental incluem os pontos de referência (LYNCH, 1960), os quais exercem uma regra importante na estruturação da informação espacial e formação do mapa cognitivo (SORROWS; HIRTTLE, 1999; BURNETT; LEE, 2005; SINGER; ABROMS; ZENTALL, 2006). Segundo Lynch (1960), os pontos de referência são utilizados pelos observadores para se locomoverem entre diferentes localizações ou para fornecerem instruções do espaço a outro (ex.: edifícios, parques, torres).

Com o intuito de aumentar a usabilidade dos SINGRA, em termos de eficiência, eficácia e satisfação por parte dos usuários, os pesquisadores têm apresentado recomendações de projeto para incluir pontos de referência específicos (BURNETT et al., 2001; ROSS; MAY; BAYER, 2004a; ROSS; MAY; GRIMSLEY, 2004b; MAY; ROSS; OSMAN, 2005a). De um número potencial de pontos de referência, o semáforo tem sido o elemento mais valorado, seguido dos viadutos (BURNETT et al., 2001, MAY; ROSS; BAYER, 2003; MAY; ROSS; OSMAN, 2005a; PUGLIESI et al., 2014).

Em uma pesquisa realizada por pesquisadores da Universidade de Nottingham, Inglaterra, os pontos de referência que fazem parte do 'mobiliário viário' (semáforo, travessia de pedestre e posto de combustível) foram destacados como sendo os mais importantes na navegação em automóvel (BURNETT et al., 2001). No entanto, os autores afirmam que os resultados são genéricos e os melhores pontos de referência podem ser escolhidos caso a caso.

Considerando os aspectos subjetivos da usabilidade de sistema, os estudos têm indicado que a qualidade do ponto de referência pode ser um fator que influencia positivamente o desempenho e a segurança do motorista (BURNETT 2000; BURNETT et al., 2001; LABIALE, 2001; BURNETT; PORTER 2002; ROSS; MAY; BAYER, 2004a; ROSS; MAY; GRIMSLEY, 2004b; MAY; ROSS; OSMAN, 2005a; MAY; ROSS; BAYER, 2005b; PUGLIESI; DECANINI; TACHIBANA, 2009).

Neste sentido, os pesquisadores têm investigado 'o que' constitui a qualidade de um ponto de referência (BURNETT 1998; BURNETT et al., 
2001, ROSS; MAY; BAYER, 2004a; ROSS; MAY; GRIMSLEY, 2004b). Diversos atributos têm sido sugeridos pela literatura, os quais são caracterizados em termos de atratividade (visual, semântica e estrutural) (BURNETT, 1998; SORROWS; HIRTLE, 1999; BURNETT et al., 2001; RAUBAL; WINTER, 2002).

Entretanto, no que se refere aos tipos de pontos de referência e aos atributos que definem a qualidade desses elementos que são recomendados para navegação em automóvel, não se pode afirmar que os resultados advindos de estudos conduzidos com motoristas tendo visão normal de cores (VNC) podem ser estendidos para motoristas com deficiência na visão de cores (DVC).

Para identificar pontos de referência que possam auxiliar a navegação de motoristas que apresentam deficiência na visão de cores, este trabalho de pesquisa chama a atenção para três questões. A primeira é verificar se a utilização dos diferentes pontos de referência, pelas pessoas que possuem deficiência na visão de cores, acontece na mesma proporção. A segunda é conhecer se os motoristas que possuem deficiência na visão de cores utilizam, em proporções similares, os mesmos tipos de pontos de referência que são utilizados pelos motoristas que apresentam visão normal de cores. A terceira é saber quais atributos melhor indicam a qualidade de um ponto de referência de modo que seja facilitada a seleção desses para inclusão em sistemas de navegação em automóvel, voltados para motoristas com DVC.

\section{Método ${ }^{5}$}

O método de trabalho adotado nesta investigação consistiu na seleção da área de estudo, na determinação de procedimentos de realização do experimento e na extração e organização dos dados coletados, de modo que

\footnotetext{
5 O número do processo junto ao Comitê de Ética em Pesquisa é 11242212.6.0000.5402, com data de aprovação em 14/12/2012
} 
fossem idênticos àqueles empregados por Pugliesi et al. (2014). Desta maneira, foi possível comparar dados sobre a frequência de utilização dos pontos de referência entre os dois grupos de motoristas do gênero masculino, com base nos diferentes tipos de visão de cores de ambos, um com deficiência na visão de cores e outro com visão normal de cores.

Este trabalho leva em consideração tanto os tricromátas anômalos com grau de anomalia elevada, bem como os dicromátas, ambos com deficiência no 'cone verde' ou no 'cone vermelho', conforme classificação apresentada por Rigden (1999). O fato de ser reprovado no teste que avalia a presença de deficiência na visão de cores implica em algumas das condições citadas anteriormente, portanto, estar em não apto a participar dos experimentos.

Quanto às técnicas de externalização de informação, foram empregadas o esboço cartográfico e as anotações verbais, a fim de contemplar indivíduos que se apresentem com diferentes estilos cognitivos, conforme recomenda a literatura (CYBIS; BETIOL; FAUST, 2010). Neste estudo, a determinação da qualidade de um ponto de referência faz utilização de atributos que se encontram disponíveis nos três modelos que foram determinados por Burnett (1998), Burnett et al., (2001) e Raubal (2002). Os atributos foram organizados de acordo com o tipo de atratividade: visual (cor, área da fachada, visibilidade, textura, permanência, notabilidade, previsibilidade em localização, familiaridade, previsibilidade em aparência, unicidade) e semântica (importância histórica e cultural, sinais e marcas explícitas, significados implícitos).

\section{1 Área de estudo}

Foi selecionada uma área em Presidente Prudente (cidade com pouco mais de 200 mil habitantes), localizada no Estado de São Paulo, e é conhecida pelas pessoas que participaram do experimento (Figura 1). Foram selecionados quatro locais para fazer parte da rota de estudo. O primeiro e o quarto ponto foram a origem e o destino, respectivamente. Dois pontos 
intermediários foram estabelecidos e se encontram em regiões bastante conhecidas pelos participantes.

Como ponto inicial foi determinada a entrada principal do campus da FCT/UNESP e como ponto final a igreja Nossa Senhora Aparecida, localizada depois da passagem em um viaduto sobre uma ferrovia, atualmente desativada. Os pontos intermediários foram a principal estação rodoviária interurbana e a igreja matriz, Catedral de São Sebastião. A escolha dos pontos intermediários aconteceu para forçar a seleção de uma rota que pudesse contemplar um número variado de pontos de referência. Entre cada um dos locais, há vários elementos, dentre os quais destacam-se diversos semáforos.

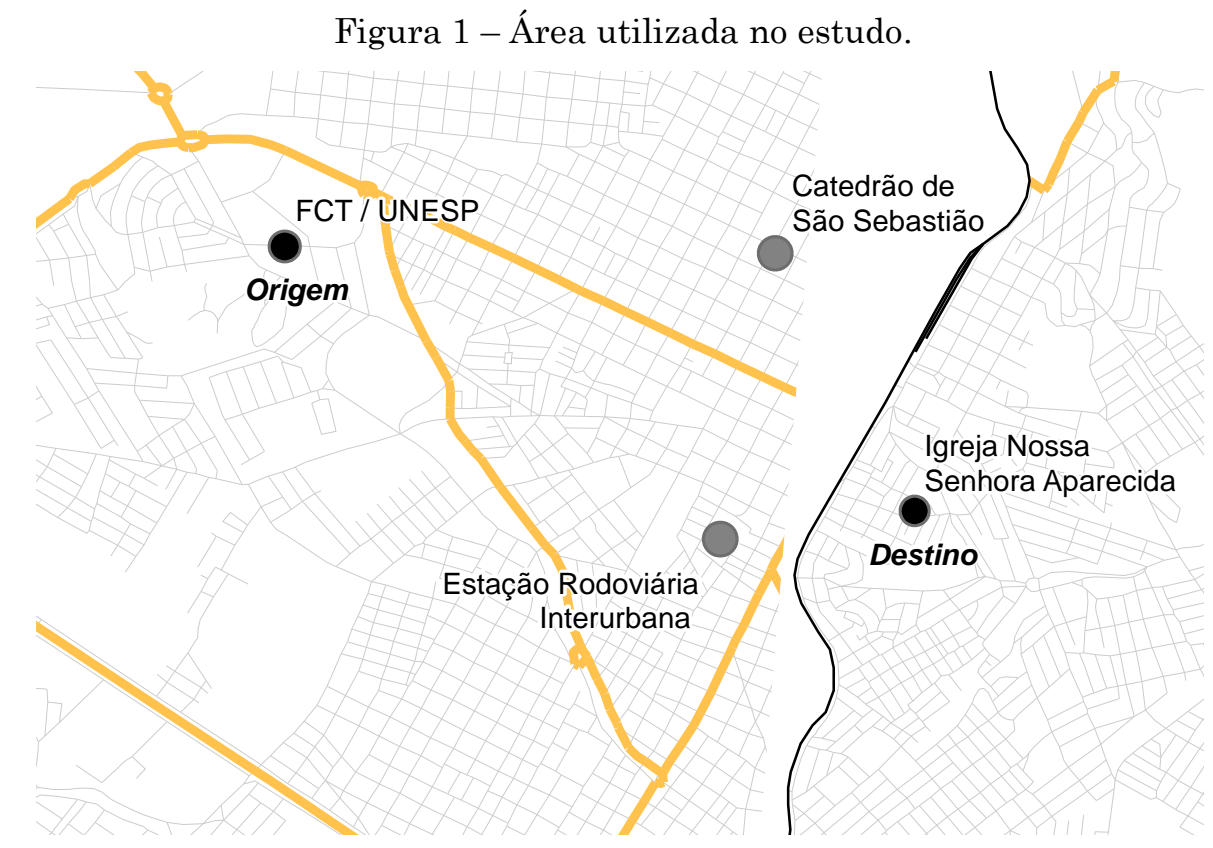

Fonte: elaborada pelos autores

\subsection{Participantes}

Um total de 15 homens com DVC participou deste experimento, com média de idade igual a 34,5 (desvio-padrão $=14,41$ e intervalo de 19 a 59). Os participantes possuíam tempo de carteira de habilitação com média de 15,35 anos (desvio padrão $=14,65$ e intervalo entre 01 e 41 anos). $O$ recrutamento foi feito de quatro maneiras: convidando pessoalmente as pessoas que já se 
conhecia ter deficiência na visão de cores; via email FCT-TODOS, do campus da Unesp de Presidente Prudente; via Facebook; e com fixação de cartazes pelo campus. Um total de três requisitos básicos foi utilizado na seleção do participante: possuir DVC, ter experiência com direção de automóvel e possuir "boa familiaridade" com a cidade de Presidente Prudente.

\subsection{Procedimentos}

A entrevista aconteceu individualmente. Os locais de entrevistas variaram de acordo com a disponibilidade do participante. Alguns foram entrevistados em suas residências, outros em seus locais de trabalho, além daqueles que estiveram disponíveis em diferentes locais do campus da FCTUNESP. Antes de iniciar o experimento, o participante foi esclarecido novamente sobre o objetivo da pesquisa, leu e assinou o Termo de Consentimento Livre e Esclarecido. Então, um questionário de caracterização foi aplicado para obter dados pessoais sobre idade, tempo de direção, experiência com mapas e desenhos, os quais pudessem apoiar as análises, caso houvesse necessidade. Depois disto, o Teste de Ishihara (ISHIHARA, 1972) foi aplicado para confirmar a presença de DVC. De 16 homens recrutados, um candidato foi excluído porque conseguiu acertar a maior parte das placas.

Em seguida, foi feita utilizado um roteiro que orientou o participante a fazer um esboço cartográfico (map sketch) e uma anotação verbal (verbal note) que contemplasse uma rota, conforme descrito na seção ‘área de estudo'. Em ambas as atividades foi solicitado a inserção de todos os elementos que considerasse necessários para serem utilizados por outro motorista com DVC e que desconhecesse completamente a área.

Com o intuito de aumentar a seriedade nas respostas, foi reforçado que o desenho e o roteiro seriam utilizados posteriormente por um motorista totalmente não familiarizado com a área. Então, foi solicitado que considerasse os pontos de origem e destino, tendo que passar, 
obrigatoriamente, pelos intermediários. Para produzir os esboços, foram utilizados um lápis e uma folha de papel de tamanho A4. O lápis foi utilizado para fazer o esboço cartográfico e a anotação verbal sobre a folha e para responder aos questionários.

Depois de concluído o desenho, solicitou-se também que o participante colocasse uma nota para cada ponto de referência presente em seu desenho, em que o menor valor está associado à baixa relevância (1) e o maior valor (9) à alta relevância. Esta etapa teve a finalidade de coletar informação sobre a qualidade dos pontos de referência que foram utilizados pelos motoristas. Para produzirem as anotações verbais, foi solicitada a escrita de um texto que descrevesse a mesma rota com todos os elementos que considerasse necessários. Alguns participantes parecem utilizar poucos pontos de referência, possivelmente, devido à dificuldade de desenhar (REIS, 2010). O emprego de anotações verbais neste trabalho de pesquisa foi feito com o intuito de verificar se haveria algum objeto não apontado no esboço e que ainda fosse importante para a navegação.

O tempo da tarefa, o tipo e a quantidade de pontos de referência que poderiam ser utilizados não foram controlados; foram livres de modo a evitar tendências, conforme recomendações da literatura (BURNETT, 1998; BURNETT et al.; 2001). Isto permitiu que o participante elencasse tudo o que considerava necessário para que outra pessoa fizesse o uso de modo que considerasse o mais apropriado possível. Cada experimento durou cerca de 40 a 60 minutos. Esse tempo relativamente longo aconteceu devido ao número elevado de questionários respondidos, além das duas tarefas principais as quais consistiram em fazer um esboço da rota e fazer uma descrição do roteiro.

\subsection{Organização dos dados}

O presente trabalho de pesquisa utiliza a quantificação objetiva dos pontos de referência utilizados e a classificação subjetiva sobre a qualidade desses elementos. A abordagem utilizada para extrair e organizar os pontos 
de referência foi similar aos trabalhos de Alm (1990), Obata, Daimon e Kawashima (1993), Burnett (1998) e Pugliesi, Decanini et al. (2014). Os pontos correspondentes à origem (universidade), destino (igreja) e intermediários (estação rodoviária e igreja matriz) não foram considerados nas análises. Um exemplo de esboço cartográfico elaborado por um participante com DVC é mostrado na Figura 2, e a anotação verbal de outro participante encontra-se exibida na Figura 3.

Figura 2 - Área utilizada no estudo

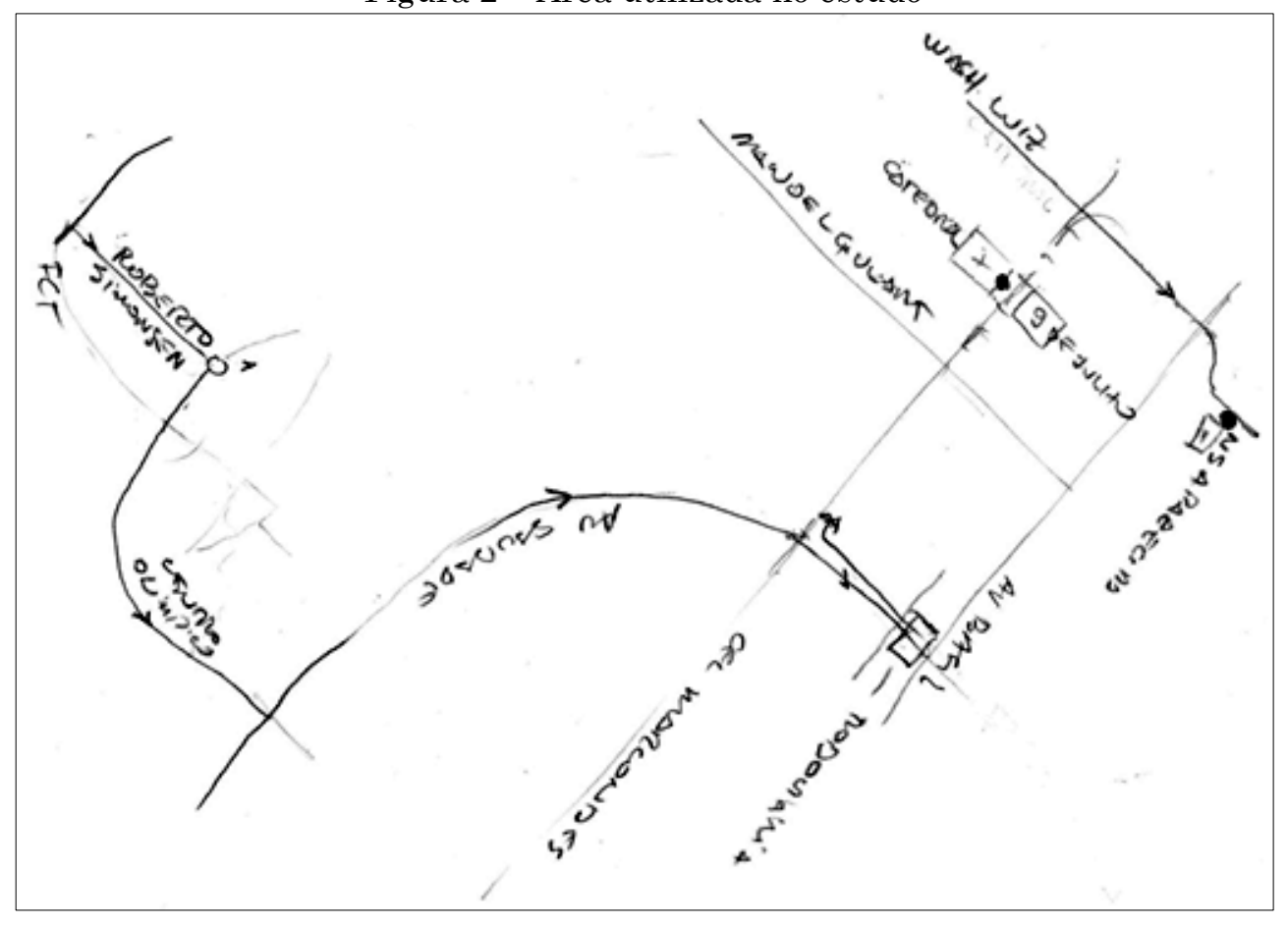

Figura 3 - Área utilizada no estudo 


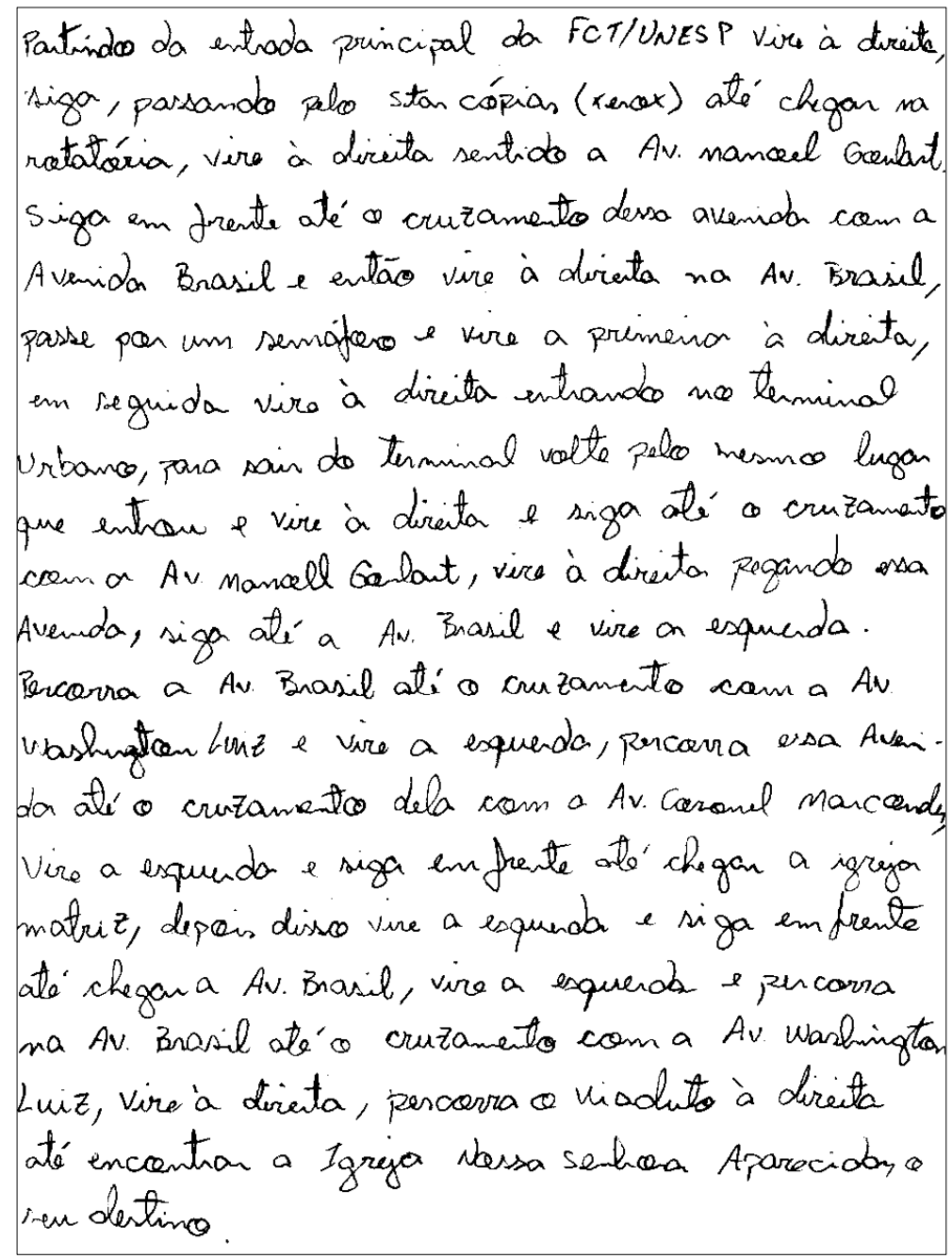

Fonte: Elaborada pelos autores.

Os pontos de referência obtidos neste trabalho foram tabulados em paralelo com alguns dos pontos obtidos no trabalho de Pugliesi et al. (2014), considerando-se somente os participantes do gênero masculino daquele estudo. Uma vez que a área de estudo e o método de trabalho é similar em ambos os trabalhos de pesquisa foi possível verificar se os motoristas que possuem deficiência na visão de cores utilizam ou não, na mesma proporção, os pontos de referência utilizados pelos motoristas que apresentam visão normal de cores.

Bos (1984) classifica os símbolos cartográficos quanto à dimensão espacial (ponto, linha e área) e quanto à forma (geométrica, pictórica e alfanumérica). Fairbain (1993) aponta que os textos são considerados o quarto 
tipo de símbolo cartográfico e classifica os textos na Cartografia em toponímicos (descreve o nome do objeto) e não toponímicos (indica outra característica do objeto). Desta maneira, os pontos de referência presentes nos esboços cartográficos foram reconhecidos como símbolos pontuais geométricos, pictóricos e textuais. No caso das anotações verbais, os pontos de referência foram identificados tanto pelo seu nome quanto pela sua função. Dentre os textos não toponímicos, Fairbain (1993) classifica como funcionais uma parte daqueles que não são usados para identificar os objetos (ex.: Centro de Esporte; Posto de Resgate). Foi empregada a abordagem de quantificação objetiva dos pontos de referência utilizados, seguindo abordagens conduzidas em outras investigações (ALM, 1990, OBATA, DAIMON; KAWASHIMA, 1993; DAIMON et al., 2000; LEE; FORLIZZI; HUDSON, 2008; PUGLIESI et al., 2014).

Para determinar os atributos que mais explicam a importância dos pontos de referência na rota da área de estudo, foi utilizada a indicação sobre a classificação subjetiva, conforme recomenda a literatura (BURNETT, 1998, SORROWS; HIRTLE, 1999; BURNETT et al., 2001; RAUBAL; WINTER, 2002). Foi feito o cálculo da média ponderada de cada atributo, de modo que quanto maior o número de pontos de referência da mesma categoria entre os participantes, maior fosse o peso atribuído àquele elemento, independentemente da quantidade de uso pelo mesmo participante. Foi fixado o número de pontos de referência em função do número de pessoas que utilizou e não da quantidade de vezes que apareceu em todos os esboços.

A análise dos dados sobre os atributos que estão relacionados à qualidade dos pontos de referência foi feita com o cálculo da média. Os demais dados coletados foram analisados por meio de técnicas estatísticas não paramétricas. Nas análises adotou-se um nível de significância menor ou igual a 0,05 , ou seja, um nível de confiança maior ou igual a 95\%. Para obter o p-valor em cada análise, foi utilizado nas análises o software Statistical Package for the Social Sciences, o qual se encontra disponível na FCTUNESP. 


\section{Análise dos resultados}

2.1 Proporção dos pontos de referência utilizados pelos indivíduos com DVC

Uma análise estatística foi conduzida para verificar se há predominância no uso de alguns pontos de referência usados nos esboços cartográficos pelos motoristas com DVC, considerando somente o número de pessoas que utilizou (Figura 4). Aplicou-se o teste não-paramétrico $Q$ de Cochran, também conhecido como prova $\mathrm{Q}$ de Cochran para $\mathrm{K}$ amostras relacionadas (CONOUVER, 1999). O Teste de Cochran é um procedimento usado para testar se as proporções entre três ou mais variáveis dicotômicas são iguais na mesma população (CONOUVER, 1999).

Figura 4 - Pontos de referência usados nos esboços cartográficos pelos motoristas com DVC

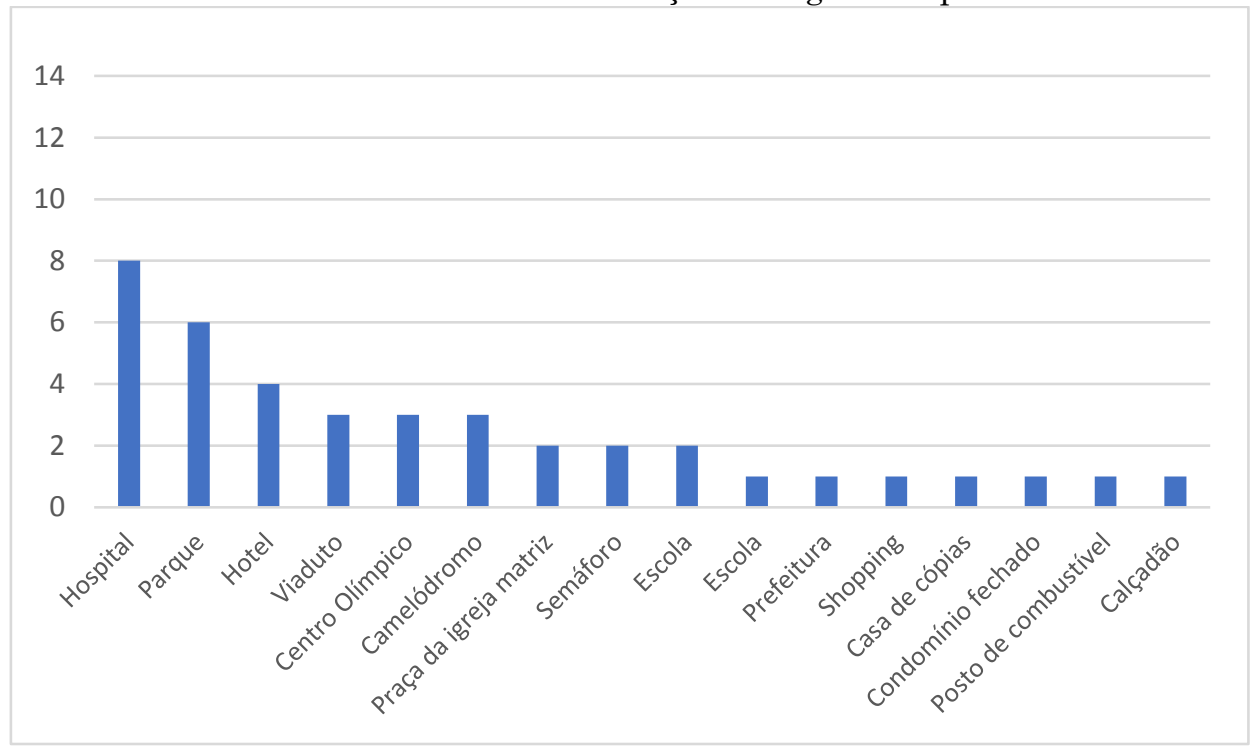

Fonte: Elaborada pelos autores.

O teste foi aplicado em quatro etapas, com base na utilização dos pontos de referência em seus esboços cartográficos (Figura 4). Por exemplo, na primeira etapa, todos os pontos foram considerados, ou seja, $\mathrm{F} 0 \geq 1$ e $\mathrm{K}=16$, enquanto na última etapa somente hospital, parque e hotel participaram da análise porque levou-se em consideração frequência maior ou igual a 4, ou seja, $\mathrm{F} 0 \geq 4$ e $\mathrm{K}=3$. Os resultados indicam que nas três primeiras etapas, o 
resultado do Teste $Q$ de Cochran indica que não há qualquer diferença entre cada uma das proporções analisadas (Tabela 1). Mas, que na quarta etapa, o hospital, o parque e o hotel foram usados na mesma proporção.

Tabela 1 - Resultados das análises que verificam se há diferenças entre as proporções nos esboços

\begin{tabular}{c|c|c|c|c|c}
\hline Etapas & $\begin{array}{c}\text { Frequência } \\
\text { observada }\left(\mathrm{F}_{\mathrm{o}}\right)\end{array}$ & $\begin{array}{c}\mathrm{K}\left(\mathrm{n}^{\mathrm{o}} \text { de pontos de }\right. \\
\text { referência })\end{array}$ & $\begin{array}{c}\text { Graus de } \\
\text { liberdade }\end{array}$ & $\begin{array}{c}\text { Q de } \\
\text { Cochran }\end{array}$ & P-valor \\
\hline 1 & $\mathrm{~F}_{0} \geq 1$ & 16 & 15 & 58,714 & 0,000 \\
\hline 2 & $\mathrm{~F}_{0} \geq 2$ & 9 & 8 & 34,579 & 0,000 \\
\hline 3 & $\mathrm{~F}_{0} \geq 3$ & 6 & 4 & 18,429 & 0,002 \\
\hline 4 & $\mathrm{~F}_{0} \geq 4$ & 3 & 2 & 6,000 & 0,05 \\
\hline
\end{tabular}

Fonte: Elaborada pelos autores.

2.2 Frequência de uso dos pontos de referência nos esboços cartográficos entre DVC e VNC

Procurando compreender se os motoristas que possuem DVC utilizam os pontos de referência na mesma proporção que os motoristas com VNC, os pontos de referência apresentados nos esboços cartográficos deste estudo foram comparados com os pontos de referência apresentados em Pugliesi et al. (2014) em que utilizaram esboço cartográfico na coleta dos dados. Para tornar viável as análises com a estatística não paramétrica, foram selecionados os elementos que tiveram frequência de uso maior ou igual a três em pelo menos um dos grupos (Figura 5).

O teste exato de Fisher foi aplicado para comparar a frequência de uso dos pontos de referência entre os dois grupos de motoristas (DVC versus VNC). Os resultados das análises estatísticas indicam que não há diferença significativa entre os dois grupos de motoristas quanto ao uso de hospital (p $=0,5)$, parque $(p=0,5)$, centro olímpico $(p=0,213)$, viaduto $(p=0,5)$, escola $(p$ $=0,5)$, posto de combustível $(\mathrm{p}=0,299)$ e hotel $(\mathrm{p}=0,5)$. Nesta análise, os semáforos foram muito menos usados por pessoas com DVC do que por pessoas com VNC $(p=0,000)$. 
Figura 5 - Pontos de referência usados nos esboços cartográficos - diferentes visões de cores

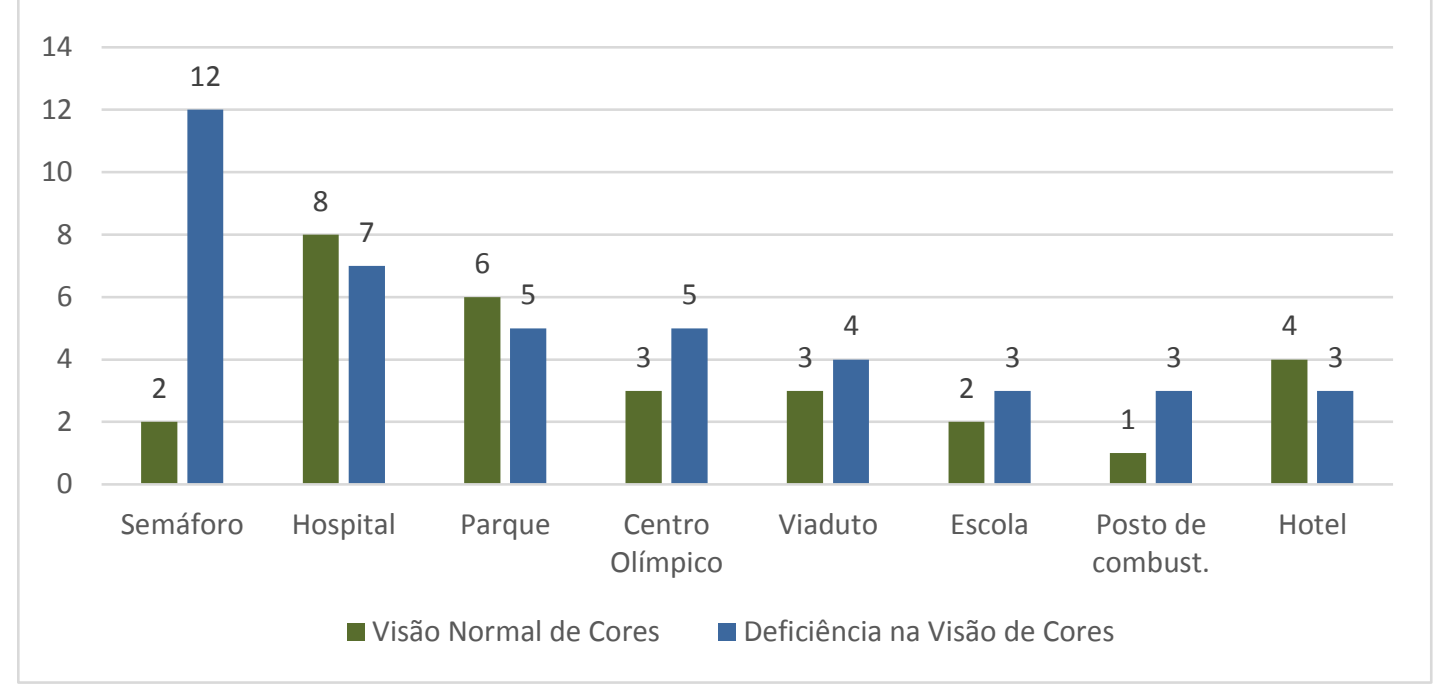

Fonte: Elaborada pelos autores.

2.3 Frequência de uso dos pontos de referência nos esboços e nas anotações pelos indivíduos com DVC

Comparando os dados presentes no gráfico da Figura 4 com os dados elencados na Figura 6, verifica-se que as diferentes técnicas de externalização de informação (esboço cartográfico e anotações verbais) apresentam variação nos tipos de pontos de referência e na frequência de utilização, entre os participantes. O teste exato de Fisher foi aplicado para verificar se há diferença estatisticamente significativa no uso de pontos de referência para a frequência maior ou igual a quatro, em pelo menos uma das técnicas.

Os resultados indicam que a utilização de hospital $(p=0,132)$, parque $(\mathrm{p}=0,107)$, hotel $(\mathrm{p}=0,326)$ e semáforo $(\mathrm{p}=0,195)$ não tiveram diferença estatisticamente significativa entre esboço cartográfico e anotação verbal. Por outro lado, o uso de viaduto se mostrou estatisticamente diferente entre as técnicas $(\mathrm{p}=0,013)$. Além do mais, considerando as anotações verbais, o semáforo foi utilizado por um terço do grupo de 15 indivíduos com DVC.

Figura 6 - Frequência de uso dos pontos nas anotações verbais - DVC 


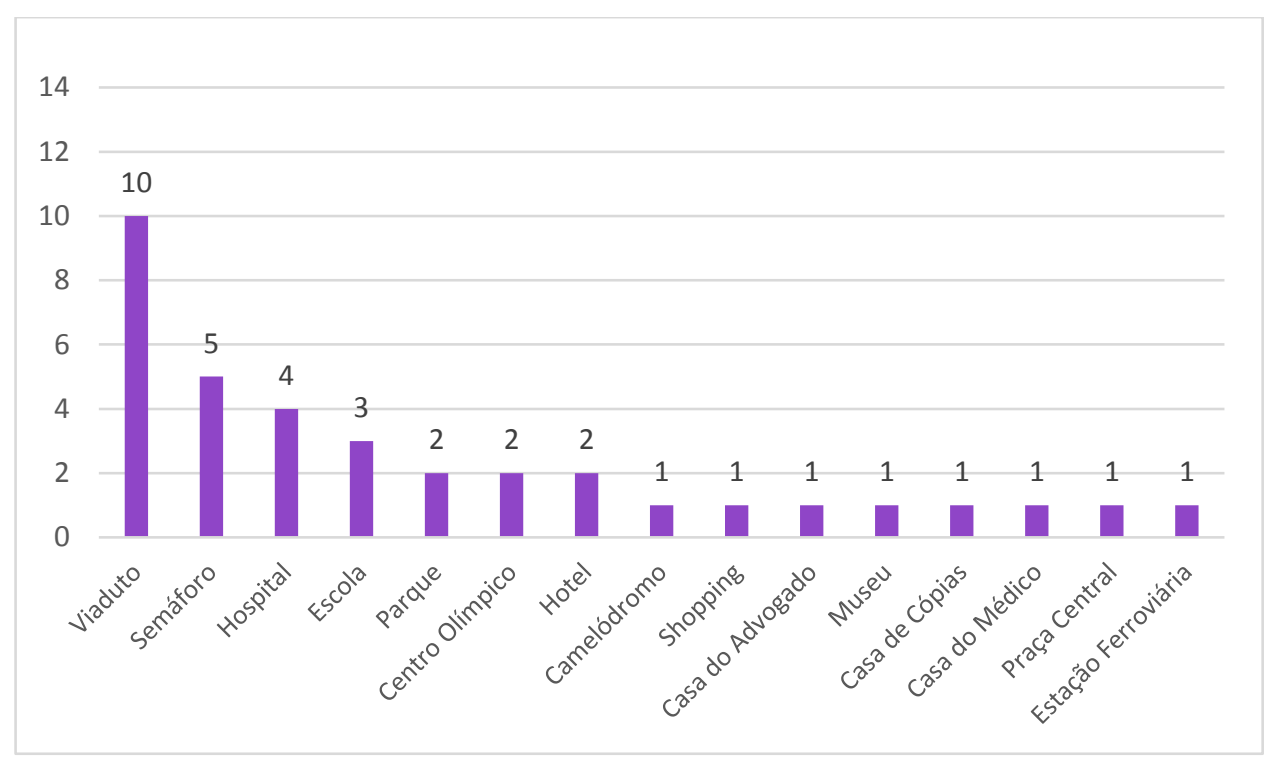

Fonte: Elaborada pelos autores.

2.4 Qualidade dos pontos de referência

Uma análise foi feita para compreender o que pode caracterizar a qualidade dos pontos de referência que foram utilizados pelos motoristas com DVC, como informação de guia de rota em automóvel, considerando a área do estudo na cidade de Presidente Prudente, SP. Para isto, foram utilizados os dados obtidos a partir dos pontos de referência elencados nos esboços cartográficos, em que cada participante marcou uma nota para cada atributo de cada elemento utilizado.

A média ponderada de cada atributo para o conjunto de pontos de referência foi utilizada. No caso dos atributos visuais, considerando o intervalo de 1 a 9 , os valores acima de 6 (25\%), são destacados para permanência, notabilidade, previsibilidade em aparência, unicidade, familiaridade, visibilidade e previsibilidade em localização (Figura 7). Por outro lado, os menores valores, abaixo de 6 , foram atribuídos à cor, área da fachada, forma e textura. Com relação aos atributos correspondentes à atratividade semântica, embora nenhum deles teve destaque, a qualidade de maior relevância esteve relacionada à importância histórica e cultural (Figura 7). 
Figura 7 - Atratividade dos pontos de referência para indivíduos com DVC

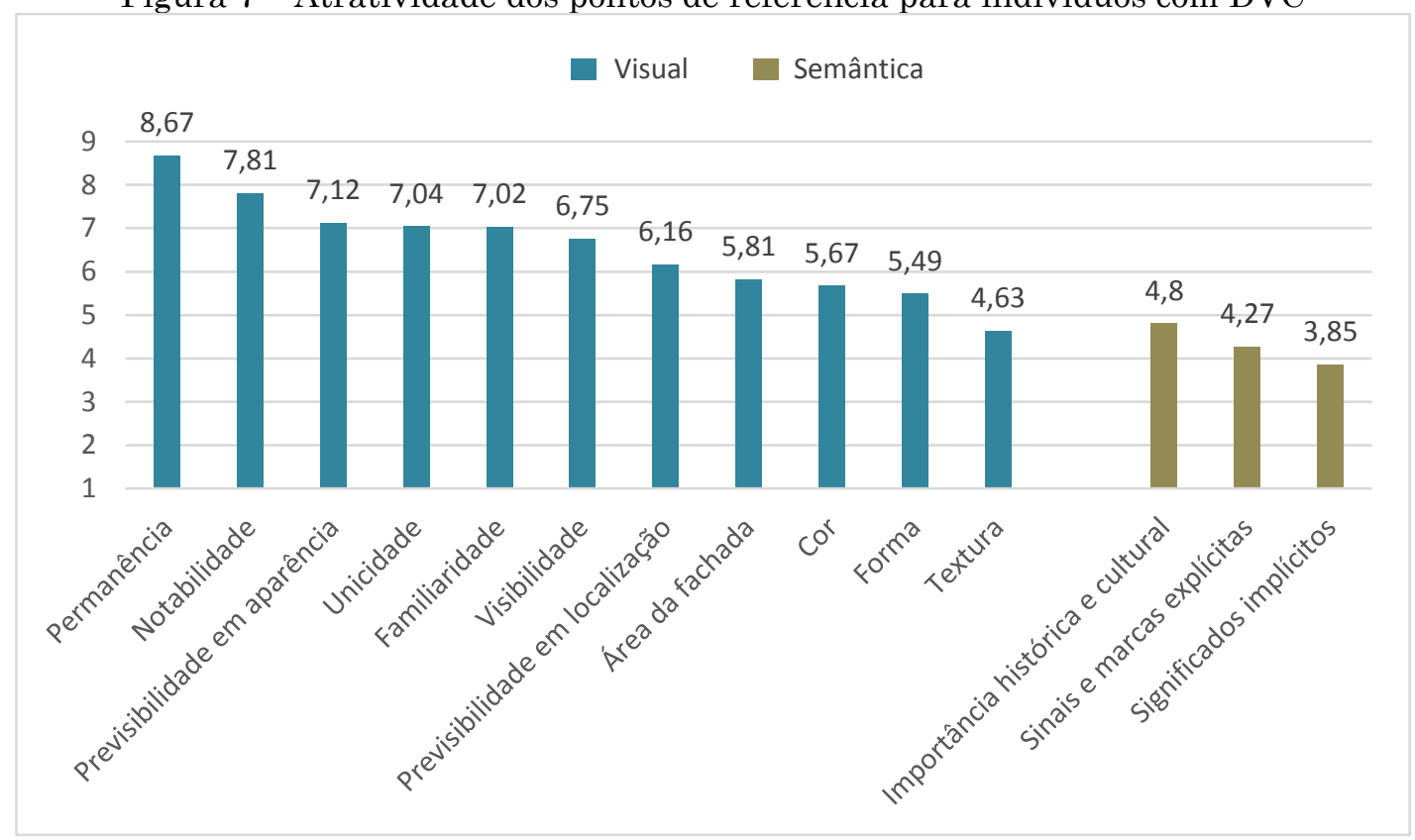

Fonte: Elaborada pelos autores.

\section{Discussões e considerações finais}

A partir das análises efetuadas sobre os dados coletados nos esboços cartográficos, conclui-se que os motoristas com DVC não utilizaram todos os pontos de referência na mesma proporção, permitindo levar à compreensão de que alguns são mais importantes que outros. Os três elementos mais utilizados nos esboços e que aparecem na mesma proporção foram hospital, parque e hotel. Este achado apresenta diferenças com os resultados encontrados na pesquisa realizada por Pugliesi et al. (2014), a qual considerou somente motoristas que possuem VNC e os semáforos foram utilizados por 12 dos 15 homens. Neste trabalho de pesquisa, o parque indicado pelos participantes (chamado de Parque do Povo) tem a característica de ser atrativo visualmente, é grande em extensão e é importante para atividades de lazer da população.

Quanto à utilização de pontos de referência pelos indivíduos com DVC e considerando diferentes técnicas de externalização, os semáforos foram mais 
utilizados nas anotações verbais do que nos esboços cartográficos, numa proporção de 5 para 2. Isto indica que os motoristas com DVC também podem usar o semáforo como elemento de informação nos sistemas de guia de rota. Estes resultados indicam que a técnica de anotação verbal complementou a técnica de esboço cartográfico. Por outro lado, hospital, viaduto e posto de combustível foram elementos que não tiveram diferenças quando analisadas as diferenças entre as duas técnicas. Então, uma análise refinada foi feita para compreender melhor esta diferença.

Diferente dos resultados apresentados nos esboços cartográficos, a técnica de anotação verbal permitiu identificar que os viadutos foram muito utilizados, confirmando resultados encontrados na literatura que estudou os mapas cognitivos de pessoas com VNC (BURNETT et al., 2001, MAY; ROSS; BAYER, 2003; MAY; ROSS; OSMAN, 2005a). No estudo atual, ao combinar os resultados obtidos nas técnicas de esboço cartográfico e anotação verbal, viaduto e hospital foram os mais utilizados pelos indivíduos com DVC.

Independente da técnica empregada para externalizar os dados, hospital, viaduto e semáforo foram utilizados por pelo menos um terço dos participantes. Esses pontos de referência se destacam visualmente (hospital) e estruturalmente (viaduto e semáforo), sugerindo que os atributos estruturais apontados por Burnett et al. (2001) e Raubal e Winter (2002) possam ser analisados em futuras investigações. Os termos 'semáforo' e 'viaduto' se apresentam com brevidade no número de caracteres, fatores que podem torna-los importantes de serem reproduzidos verbalmente, conforme apontado por Burnett et al. (2001).

Apesar dos resultados encontrados sugerirem que hospitais sejam importantes, é necessário que os sistemas de navegação façam o cálculo do ângulo de visibilidade que o motorista tem para ver o ponto de referência, e isto pode ser feito conforme técnica sugerida por Raubal e Winter (2002). Então, a exibição de hospitais seria feita a partir de outros fatores que deveriam ser levados em consideração, conjuntamente. 
Com relação ao nível de qualidade atribuído aos pontos de referência pelos motoristas com DVC, destaca-se que o elemento visual mais valorado foi a permanência, independente de se apresentarem com logotipos (ex.: símbolo) ou marcas (ex.: cores ou outros padrões gráficos). Sequencialmente, aparecem: notabilidade, previsibilidade em aparência, unicidade, familiaridade, visibilidade e previsibilidade em localização (correspondente aos valores iguais ou maiores que 6 (dentro do primeiro intervalo de $25 \%$ ). Por outro lado, os menos importantes (considerados abaixo do valor igual a 6) seguiram na seguinte sequência: cor, área da fachada, forma e textura. Ainda assim, os atributos visuais se demonstram mais importantes do que os atributos cognitivos. Neste estudo, não foram utilizados todos os atributos elencados por Burnett et al. (2001); portanto, novas investigações podem incluí-los.

Os pontos de referência elencados em ambas as técnicas utilizadas nesta pesquisa podem ser vistos com certa facilidade no mundo real. Isto pode ser compreendido de modo que permita ao motorista olhar para o elemento no ambiente urbano e verificar sua presença no sistema de navegação, não importando a ordem de visualização; podendo ser primeiro no sistema de navegação e depois no mundo real, conforme apresentado no modelo concebido por Burnett (1998).

De modo geral, a baixa frequência no uso de pontos de referência pelos motoristas que participaram deste estudo parece estar relacionada com o gênero dos participantes. Pesquisas na área da cognição espacial aponta que os homens usam muito menos pontos de referência do que as mulheres (DABBS et al., 1998; BURNETT, 1998). E que isto pode estar relacionado com fatores antropológicos, de acordo com a perspectiva evolucionária de Silverman e Eals (1992) apud Dabbs et al. (1998). Também, pode estar relacionado à fatores hormonais, sendo que os maiores níveis de testosterona no organismo humano podem influenciar positivamente a habilidade espacial, característica de maior predominância no homem (HOOVEN, 2004). 
A partir do exposto, vale apontar que cada local tem sua particularidade e a área de estudo pode influenciar na quantidade, variedade e qualidade dos pontos de referência. Portanto, torna-se necessário a realização de mais estudos, em locais que se apresentam com paisagens distintas e em ambientes urbanos de diferentes portes, uma vez que os melhores pontos de referência podem ser escolhidos caso a caso.

Soares (2015) investigou o uso de pontos de referência em mapas de SINGRA junto a um grupo de motoristas com VNC e encontrou que a preferência por pontos de referência que tinham alto grau de similaridade com seus referentes no mundo real (ex.: Museu de Artes de São Paulo - MASP) foi estatisticamente significativa quando comparado com os mesmos mapas sem tais pontos de referência. Pesquisa similar pode ser conduzida com motoristas que possuem DVC para apontar recomendações de projeto que tratam da exibição de pontos de referência que se apresentam com alto nível de iconicidade, considerando a atratividade visual, principalmente com as formas, texturas e cores.

Este trabalho de pesquisa levou em conta os indivíduos com deficiência na visão de cores, tanto tricromátas anômalos com grau de anomalia elevada, bem como os dicromátas, ambos com deficiência no verde ou no vermelho. Não se consideraram tricromátas anômalos com grau de anomalia moderada ou baixa e nem os dicromátas com deficiência no cone azul. Outras investigações podem incluir estes subgrupos.

Recomenda-se que seja realizado o projeto de símbolos visuais e/ou sonoros, em especial semáforo, viaduto e parque, de modo que apoiem nas tomadas de decisão de motoristas com DVC, para a tarefa de manutenção em rota. Um projeto de mapas para motoristas daltônicos (OLIVEIRA et al., 2018) pode ser considerado na inserção tais pontos de referência. Posteriormente, deveria ser avaliada a usabilidade e a estética dessas representações, atentando para a eficiência, eficácia, satisfação e segurança do motorista. 
O tempo para concluir as tarefas foi elevado e o fator que mais influenciou isto foi a etapa de preenchimento dos questionários. Para cada ponto de referência utilizado no esboço, dois questionários (um para a atratividade visual e outro para a atratividade semântica) deveriam ser respondidos. Isto aumentou o tempo para completar as tarefas. Consequentemente, a inserção de mais atributos implicaria em levar maior tempo na realização do experimento.

\section{Agradecimentos}

Este trabalho de pesquisa foi apoiado pela Fundação de Amparo à Pesquisa do Estado de São Paulo - FAPESP, processo número 2012/14556-8. Os autores agradecem aos participantes que se dedicaram em participar voluntariamente dos experimentos.

\section{Referências}

ALM, H. Drivers' cognitive models of routes. In W. van Winsum, H. Alm, J.M. Schraggen and J.A. Rothengatter (Eds.), Laboratory and field studies on route representation and drivers' cognitive models of routes (DRIVE II V1041 GIDS, Deliverable GIDS/NAV2). Groningen, The Netherlands: University of Groningen, Traffic Research Centre. 1990. pp. 35-48.

BOS, E. S. Cartographic Symbol Design. The Netherlands, ITC, 1984. 85p.

BURNETT, G. E. "Turn right at the King's Head": Drivers' requirements for route guidance information. $\mathrm{PhD}$ Thesis. Loughborough University, UK, 1998. 341p.

BURNETT, G. 'Turn right at the traffic lights': The requirement for landmarks in vehicle navigation systems. Journal of Navigation, vol. 53, n. 3, 2000. pp. 499-510.

BURNETT, G. E.; SMITH, D.; MAY, A. J. Supporting the navigation task: characteristics of 'good' landmarks. Contemporary Ergonomics 2001 
(Proceedings of the Annual Conference of the Ergonomics Society). 2001. pp. 441-446.

BURNETT, G, E., PORTER, J. M. An Empirical Comparison of the Use of Distance Versus Landmark Information within the Human-Machine. Human Factors in Transportation, Communication, Health and the Workplace, 2002. pp. 9-12.

BURNETT, G. E.; LEE, K. The effect of vehicle navigation systems on the formation of cognitive maps. In: Underwood (Eds.). Traffic and Transport Psychology: Theory and Application. 2005. pp. 407-418.

CONOVER, W. J. Practical nonparametric statistics. New York: J. Wiley, 1999. $584 \mathrm{p}$

ISHIHARA, S. The Series of Plates Designed as a Test for Colour-Blindness. 24 Plates Edition, S. Kanehara: Tokyo. 1972. 33p.

LABIALE, G. Visual search and preferences concerning different types of guidance displays. Behaviour \& Information Technology. London: Taylor \& Francis. vol. 20, n. 3, 2001. pp. 149-158.

LEE, J.; FORLIZZI, J; HUDSON, S. Iterative design of MOVE: A situationally appropriate vehicle navigation system. International Journal of HumanComputer Studies, vol. 66, n.3, 2008. pp. 198-215.

LYNCH K. The image of the city. Cambridge, MA: MIT Press. 1960. 194p.

MAY, A.; ROSS, T.; BAYER, S. H. Drivers' Information Requirements when Navigating in an Urban Environment. The Journal of Navigation, vol. 56, 2003. pp. 89-100.

MAY, A.; ROSS, T.; OSMAN, Z. The design of next generation in-vehicle systems for the older driver. Interacting with Computers, vol. 17, n.6, 2005a. pp. 643659.

MAY, A. J.; ROSS, T.; BAYER, S. H. Incorporating Landmarks in Driver Navigation System Design: An Overview of Results from the REGIONAL Project. The Journal of Navigation, vol. 58, 2005b. pp. 47-65.

MAY, A. J., ROSS, T. Presence and Quality of Navigational Landmarks: Effect on Driver Performance and Implications for Design. Human Factors, vol. 48, n. 2, 2006. pp. 346-361. 
OBATA, T.; DAIMON T.; KAWASHIMA, H. A cognitive study of invehicle navigation systems: aplying verbal protocol analysis to usability evaluation. Proceedings of Vehicle Navigation and Information Systems Conference. Piscataway: Institute of Electrical and Electronics Engineers, 1993. pp. 232237.

OLIVEIRA, R. F.; PUGLIESI, E. A.; MARQUES, A. P. S.; DECANINI, M. M. S. Preference of Drivers with Color Vision Deficiency for Maps of In-Car Route Guidance and Navigation System. Boletim de Ciências Geodésicas, vol. 24, n. 2, 2018. pp. 186-201.

PAPINSKI, D.; SCOTT, D. M.; DOHERTY, S. T. Exploring the route choice decisionmaking process: A comparison of planned and observed routes obtained using person-based GPS. Transportation Research Part F, vol.12, n.4, 2009. pp. 347-358.

PETCHENIK, B. B. The Nature of Navigation: Some Difficult Cognitive Issues in Automatic Vehicle Navigation. IEEE Explore, Toronto, 1989. pp. 43-48.

PUGLIESI, E. A.; DECANINI M. M. S.; TACHIBANA V. M. Evaluation of the Cartographic Communication Performance of a Route Guidance and Navigation System. Cartography and Geographic Information Science, vol. 36, n. 2, 2009. pp. 193-207; April.

PUGLIESI, E. A.; REIS, Y. C.; DECANINI, M. M. S.; TACHIBANA, V. M. Drivers' Requirements for In-Car Route Guidance Information: Gender and Individual Differences. Brazilian Journal of Cartography, vol. 66, n.5, 2014. pp.681690.

RAUBAL, M.; WINTER, S. Enriching Wayfinding Instructions with Local Landmarks. In: M. Egenhofer and D. Mark (Eds.), Geographic Information Science - Second International Conference GIScience. Lecture Notes in Computer Science 2478. 2002. pp. 243-259.

REIS, Y. C. Seleção de Informações de Guia de Rota Para a Concepção de Sistemas de Navegação. Dissertação de Mestrado. Universidade Estadual Paulista - Faculdade de Ciências e Tecnologia, Presidente Prudente, 2010. $130 \mathrm{p}$.

RIGDEN, C. The eye of the beholder - Designing for colour-blind users. British Telecommunications Engineering. vol. 17. 1999. 
ROSS, T.; MAY, A.; BAYER, S. Adding value for the navigation user. Proceedings of the Nav 04 Conference, 'Location and Timing Applications', Maritime AIS and GNSS. Church House. 2004a. pp. 1-11.

ROSS, T.; MAY, A, GRIMSLEY, P. L. Using traffic light information as navigational cues: implications for navigation system design. Transportation Research Part F 7. 2004b. pp.119-134.

SINGER, R. A.; ABROMS, B. D.; ZENTALL, T. R. Formation of a Simple Cognitive Map by Rats. International Journal of Comparative Psychology, vol. 19. 2006. pp.417-425.

SOARES, J. M. M. Preferência por Marcos em Mapas 3D de Sistema de Navegação e Guia de Rota em Automóvel. Dissertação de Mestrado. Universidade Estadual Paulista "Júlio de Mesquita Filho", Programa de PósGraduação em Ciências Cartográficas, Presidente Prudente, 2015. 127p.

SORROWS, M. E.; HIRTLE, S. C. The nature of landmarks for real and electronic spaces. In C. Freksa \& D. M. Mark (Eds.). Spatial information theory Cognitive and computational foundations of geographic information science. Berlin: Springer. 1999. pp. 37-50. 